\title{
Asociaciones de poliquetos del estuario del río Gallegos, Patagonia Austral, Argentina
}

\section{Polychaete assemblages of the Gallegos river estuary, Southern Patagonia, Argentina}

\author{
Susana PITTALUGA ${ }^{1}$, Zulma LIZARRALDE ${ }^{1}$, Juan Pablo MARTIN ${ }^{2}$ \\ ${ }^{1}$ Instituto de Ciencias del Ambiente, Sustentabilidad y Recursos Naturales (ICASUR), \\ Universidad Nacional de la Patagonia Austral, Unidad Académica Río Gallegos, \\ spittaluga@uarg.unpa.edu.ar \\ ${ }^{2}$ ICASUR, Universidad Nacional de la Patagonia Austral, Unidad Académica San Julián.
}

Recibido: 26/11/2018. Aceptado: 03/04/2019

\begin{abstract}
RESUMEN
Durante la primavera de 2013 se realizaron muestreos bentónicos en cuatro sitios ubicados en ambas márgenes del estuario del río Gallegos. Se tomaron cinco muestras por nivel de marea en cada sitio siguiendo una transecta perpendicular a la costa. Se calculó la abundancia relativa de las especies, la dominancia, la riqueza específica, la equitatividad y el índice de diversidad de Shannon-Wiener. Los datos de abundancia fueron comparados mediante análisis multivariado, empleando el índice de similitud de Bray-Curtis y técnicas de ordenamiento no paramétrico. Se identificaron 14 especies de poliquetos, siendo Fabricinuda sp. la más abundante, seguido por Notocirrus lorum y Kinbergonuphis dorsalis. La abundancia, la equitatividad y la diversidad mostraron diferencias significativas entre sitios. La mayor abundancia se registró en el nivel intermareal inferior de S1, adyacente a la ciudad de Río Gallegos, debido a la presencia de Fabricinuda sp. que forma estructuras biogénicas de gran extensión, con alta densidad de organismos. En ese mismo sitio se observó la mayor riqueza específica y la menor diversidad y equitatividad. Los resultados del análisis multivariado indicaron la presencia de tres grupos principales de poliquetos, asociados a sedimentos con diferente contenido de materia orgánica y distinta composición granulométrica.
\end{abstract}

Palabras clave: organismos bentónicos; estuario; poliquetos.

\begin{abstract}
During the spring of 2013, benthic samplings were conducted in four sites located on both banks of the Gallegos river estuary. Five samples were taken per tidal level at each site following a transect perpendicular to the coast. The relative abundance of the species, the dominance, the species richness, the equitability and the diversity index of Shannon-Wiener were calculated. The abundance data were compared by multivariate analysis, using the BrayCurtis similarity index, and the nonparametric ordination technique. We identified fourteen polychaete species, being Fabricinuda sp. the most abundant, followed by Notocirrus lorum and Kinbergonuphis dorsalis. Abundance, equitability and diversity showed significant differences between sites. The highest abundance was recorded in the lower intertidal level of S1 site, next to Río Gallegos city, due to the presence of Fabricinuda sp. that forms biogenic structures of great extension with high density of organisms. In the same place, the highest
\end{abstract}


species richness and the lowest diversity and equitability were observed. The results of the multivariate analysis indicated the presence of three main groups of polychaetes, associated with sediments with different organic matter content and different granulometric composition.

Key words: benthic organisms; estuary; polychaetes.

\section{INTRODUCCION}

Los poliquetos son componentes importantes de la macrofauna bentónica por estar entre los grupos dominantes en términos de abundancia, biomasa, riqueza de especies (Gambi et al., 1998) y funcionalidad ecológica, lo que les permite una alta adaptabilidad a los diferentes hábitats (Díaz-Castaneda y Harris, 2004). El conocimiento de la fauna de poliquetos es considerado un factor importante para caracterizar los distintos hábitat bentónicos y también para realizar programas de vigilancia ambiental (Pearson y Rosenberg, 1978, Cañete et al., 2000). Con frecuencia son utilizados como indicadores de impacto antrópico ya que incluyen tanto especies sensibles como tolerantes a las perturbaciones del medio, causadas principalmente por el enriquecimiento de materia orgánica, y habitan desde zonas prístinas hasta los hábitats altamente perturbados (Pocklington y Wells, 1992; Giangrande et al., 2005; Dauvin et al., 2007).

Los primeros estudios ecológicos sobre poliquetos de ambientes marinos y estuariales se iniciaron en la costa de la provincia de Buenos Aires (Bremec 1986, Elías y Bremec 1986, Elías 1992, Elías y Ieno 1993, Martin et al. 2004, Martin y Bastida 2006, entre otros). Elías et al. (2017) reúnen la información sobre la fauna de poliquetos del Mar Argentino otorgando un perfil histórico del desarrollo de esta línea de investigación en el país. En la Patagonia austral, las investigaciones pioneras sobre comunidades bentónicas se deben a Ringuelet et al. (1962, 1963) y Ringuelet (1963) las cuales fueron realizadas a partir de 1961 desde la Estación de Biología Marina de Puerto Deseado, Santa Cruz (Bastida et al., 2007). En dichos trabajos, se describe la fauna de poliquetos de fondos blandos y rocosos de la ría Deseado. Estudios posteriores incluyen las asociaciones de los poliquetos en estudios sobre comunidades bentónicas (Lizarralde y Pittaluga., 2011; Martin et al., 2015) y la presencia de poliquetos asociados al enriquecimiento de materia orgánica (Martin et al., 2009; Pittaluga et al., 2016). Las comunidades bentónicas del ambiente intermareal del estuario del río Gallegos se distribuyen siguiendo una zonación vertical, determinada principalmente por las características del sustrato (granulometría y materia orgánica) y el nivel de marea (Lizarralde y Pittaluga, 2011, Pittaluga, 2016). Los poliquetos son el taxa más abundante, seguido de los crustáceos y los moluscos (Lizarralde y Pittaluga, 2011).

Los diversos usos y actividades antrópicas desarrolladas en el estuario (actividades turísticas, de recreación, portuarias, vertido de efluentes domiciliarios), han provocado diferentes modificaciones sobre el ambiente costero. Lizarralde et al., (2018) han señalado que la estructura de la comunidad bentónica se ha visto modificada en el nivel más bajo del intermareal, ante la presencia del poliqueto Fabricinuda sp.

El objetivo del presente trabajo fue conocer la composición y distribución de las asociaciones de poliquetos en el estuario del río Gallegos, con el fin de aportar información que contribuya a la implementación de futuros monitoreos para detectar cambios ambientales en el sitio de estudio. 


\section{MATERIALES Y MÉTODOS}

\section{1 Área de estudio}

El presente estudio se llevó a cabo en el estuario del río Gallegos ( $51^{\circ} 35^{\prime} \mathrm{S}$ y $\left.69^{\circ} 01^{\prime} \mathrm{O}\right)$ ubicado al sudeste de la provincia de Santa Cruz, Argentina (Fig. 1). Se extiende en un recorrido aproximado de $45 \mathrm{~km}$ de oeste a este, desembocando en el Mar Argentino entre Punta Loyola y Punta Bustamante. En la margen sur del estuario, se ubica la ciudad de Río Gallegos, capital de la provincia de Santa Cruz. Según su rango de mareas es clasificado como un estuario macromareal (Piccolo y Perillo, 1997), con amplitudes mareales de sicigia de hasta 12 metros. La margen sur, de escasa pendiente, está formada por antiguas planicies de mareas con predominio de sedimentos finos (fango y arena), y marismas caracterizadas por la presencia de vegetación halófita (Sarcocornia perennis). La margen norte es una zona de acantilados de hasta 150 metros de altura, afectada por la erosión de las olas (Codignotto, 1990). Algunos organismos de las comunidades bentónicas del estuario constituyen un importante ítem dentro de la dieta de aves playeras y peces de importancia comercial. El estuario cumple un rol clave como área de alimentación y descanso para numerosas especies de aves neárticas y patagónicas, albergando un porcentaje significativo de sus poblaciones biogeográficas (Ferrari et al., 2002, 2007; Lizarralde et al., 2010).

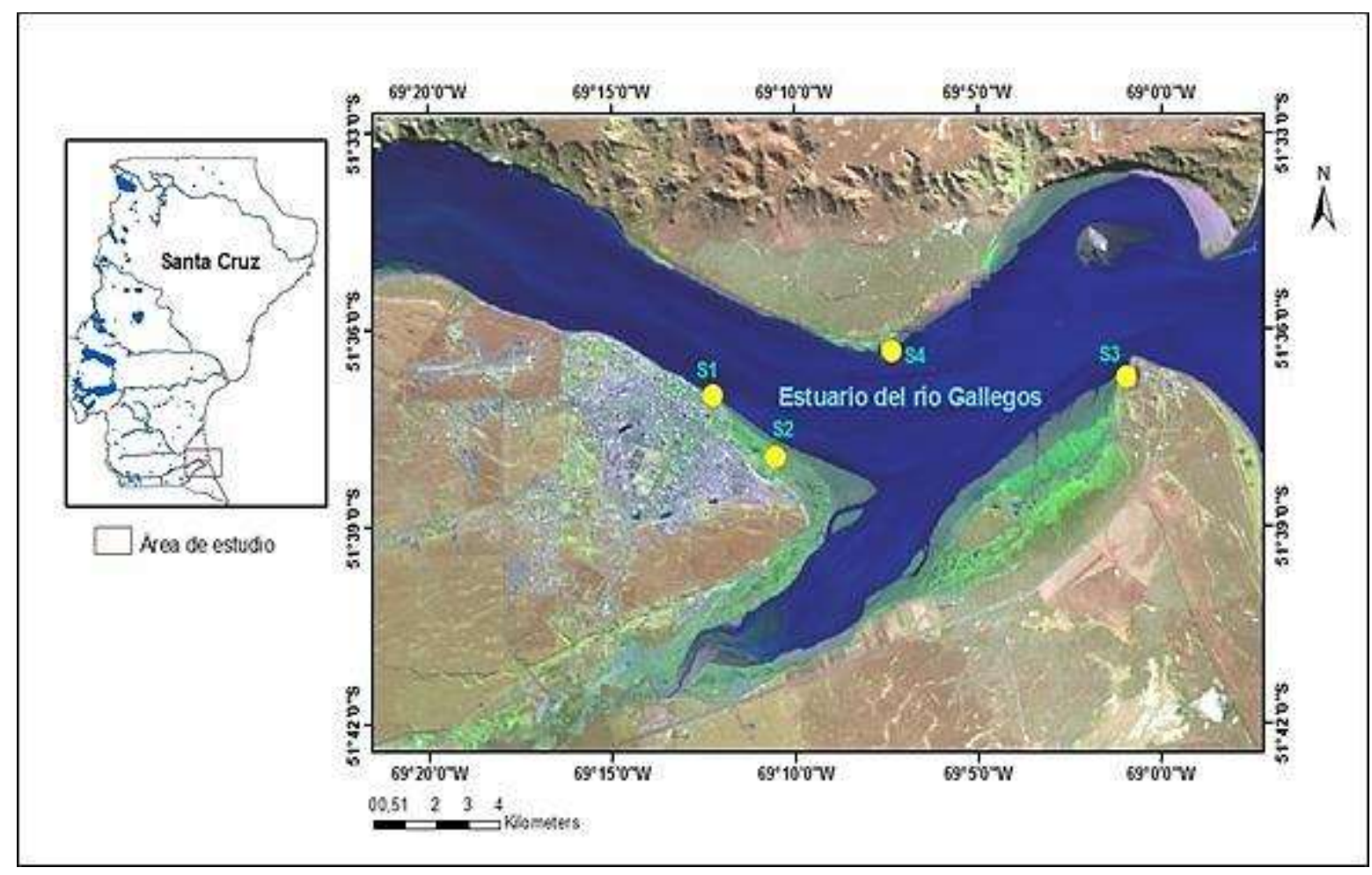

Figura 1. Área de estudio y ubicación de los sitios de muestreo: Sitio1: Costanera; Sitio 2: Emisario; Sitio 3: Punta Loyola y Sitio 4: Punta Gancho.

\subsection{Muestreo de macrofauna}

Los muestreos se efectuaron en cuatro sitios del estuario, comprendidos en un tramo de $25 \mathrm{~km}$ aproximadamente. Los mismos fueron georreferenciados y denominados como: Sitio1, Costanera; Sitio 2, Emisario; Sitio 3, Punta Loyola y Sitio 4, Punta Gancho. Los tres primeros se ubican sobre la margen sur del estuario y el último sobre la margen norte. Durante la 
primavera del 2013, se realizaron muestreos bentónicos en los niveles superior, medio e inferior del intermareal y en el superior del infralitoral. Durante la baja mar y siguiendo una transecta perpendicular a la línea de la costa, se recolectaron cinco muestras por sitio y nivel de mareas, (NI a NIV, correspondiendo el NI al nivel superior del intermareal) separados aproximadamente 500 metros entre sí, empleando un tubo ("core sampler") de $10 \mathrm{~cm}$ de diámetro enterrado $20 \mathrm{~cm}$ en el sedimento. Los organismos fueron separados utilizando un tamiz de $0,5 \mathrm{~mm}$ de abertura de malla y fijados en formol al $4 \%$, para su posterior identificación y cuantificación.

\subsection{Muestreo de sedimentos}

Para cada sitio y nivel de muestreo, se recolectaron dos muestras para el análisis de la granulometría de los sedimentos, y dos para determinar el contenido de materia orgánica, empleando un cilindro de plexiglás de $4 \mathrm{~cm}$ de diámetro. Para el análisis de granulometría las muestras de sedimento fueron secadas en estufa a $100{ }^{\circ} \mathrm{C}$ hasta peso constante, pesadas y luego separadas por tamizado en húmedo en siete fracciones correspondientes a la escala de Wentworth. Cada una de las fracciones obtenidas fueron secadas y pesadas; la fracción de sedimentos finos (menores de 0,0625 mm de diámetro) se estimó por diferencia de pesos (Gray et al. 1992). Los valores obtenidos fueron expresados como porcentajes en peso de la muestra.

El contenido de materia orgánica de los sedimentos fue determinado por pérdida de peso por ignición, siguiendo la técnica de Schulte y Hopkins (1996). Los resultados se expresaron como porcentaje en peso de la muestra.

\subsection{Análisis estadísticos}

Para describir y comparar la estructura de las asociaciones de poliquetos a partir de la información de la abundancia de especies se calculó la dominancia relativa (D), la riqueza de especies (S), el índice de diversidad específica de Shannon-Wiener (H'), y la equitatividad (J) con el índice de Pielou. Para determinar si existían diferencias significativas en la abundancia, la diversidad, la equitatividad y la riqueza de especies se aplicó una prueba no paramétrica de Kruskal-Wallis (Zar, 1996). Los datos de abundancia de las especies de poliquetos fueron analizados mediante técnicas multivariadas de agrupamiento (análisis de clúster) y de ordenamiento (escalamiento multidimensional no paramétrico o MDS). Con el objetivo de evaluar la similitud/disimilitud en la composición de las asociaciones de poliquetos entre los sitios de muestreo, se empleó el índice de similitud de Bray Curtis, previa transformación raíz cuarta de los datos. Para determinar las diferencias estadísticas en la composición específica entre los diferentes sitios, se empleó la rutina ANOSIM ("Analysis of similarities") con un nivel de significancia $\mathrm{p}<0,05$. Para evaluar el porcentaje de contribución de las diferentes especies a la similitud/disimilitud entre los sitios y niveles de muestreo, se empleó el módulo SIMPER ("Similarity percentages"). Todos los análisis se realizaron empleando el software PRIMER (Plymouth Routines in Multivariate Ecological Research) versión 6.1 (Clarke y Warwick 2001).

\section{RESULTADOS}

El análisis de la composición granulométrica de todos los sitios en conjunto, mostró que los sedimentos se caracterizaron por un alto porcentaje de sedimentos finos (limos-arcillas, arenas muy finas y finas) (Fig. 2). 
Los valores promedio de materia orgánica en los sedimentos fluctuaron dentro del intervalo de 2,23\% y 3,19\% (Fig. 3), siendo el Emisario (S2) el sitio que presentó los porcentajes más altos en el nivel superior del intermareal $(4,39 \%)$.

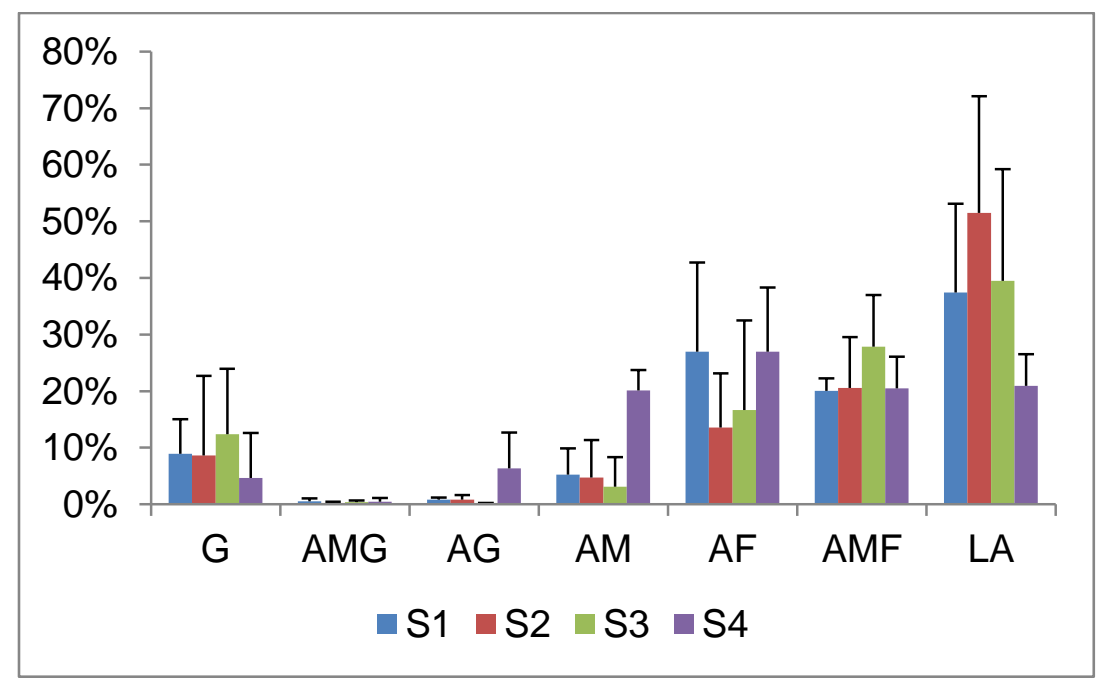

Figura 2. Composición granulométrica de los sedimentos (promedio \pm desvio estándar) por sitios. G: gravas, AMG: arenas muy gruesas, AG: arenas gruesas, AM: arenas medias, AF: arenas finas, AMF: arenas muy finas, LA: limos-arcillas. S1: Costanera. S2: Emisario, S3:

Punta Loyola y S4: Punta Gancho.

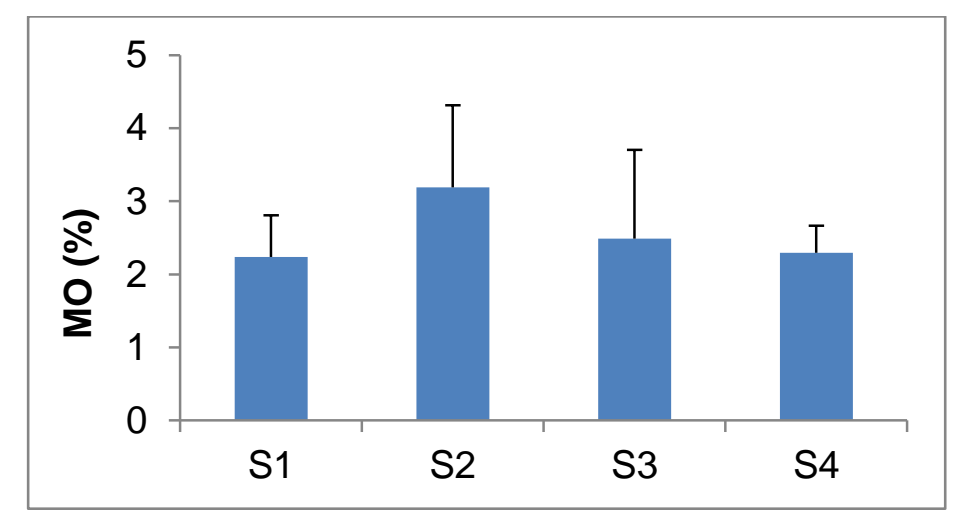

Figura 3. Porcentaje de materia orgánica (promedio \pm desvío estándar) para los diferentes sitios de muestreo. S1: Costanera. S2: Emisario, S3: Punta Loyola y S4: Punta Gancho.

Se identificaron 14 especies de poliquetos pertenecientes a 12 familias. Todas las familias estuvieron representadas por una sola especie a excepción de Onuphidae (2 spp.). Las familias que presentaron el mayor número de individuos fueron Fabriciidae, Onuphidae y Phyllodocidae, (Tabla 1). 
Tabla 1. Listado de especies de poliquetos del estuario del río Gallegos, y porcentaje de dominancia.

\begin{tabular}{|l|l|c|}
\hline \multirow{2}{*}{ Familia } & Especie & Dominancia (\%) \\
\hline Fabriciidae & Fabricinuda sp. & 87,77 \\
\hline \multirow{2}{*}{ Onuphidae } & Notocirrus lorum & 2,70 \\
\cline { 2 - 3 } & Kinbergonuphis dorsalis & 2,51 \\
\hline Lumbrineridae & Lumbrineris cingulata & 1,49 \\
\hline Phyllodocidae & Eteone sculpta & 1,39 \\
\hline Goniadidae & Glycinde armata & 1,14 \\
\hline Nephtydae & Aglaophamus praetiosus & 1,00 \\
\hline Orbiniidae & Leodamas cirratus & 1,00 \\
\hline Spionidae & Scolecolepides uncinatus & 0,66 \\
\hline Maldanidae & Clymenella minor & 0,13 \\
\hline Glyceridae & Hemipodus patagonicus & 0,12 \\
\hline Nereididae & Ceratocephale sp. & 0,04 \\
\hline Goniadidae & Ophioglycera eximia & 0,03 \\
\hline Travisiidae & Travisia sp. & 0,02 \\
\hline
\end{tabular}

La abundancia, la equitatividad y la diversidad mostraron diferencias significativas entre sitios (Test de Kruskall-Wallis $\mathrm{p}<0,05$ ) (Fig. 4). La mayor diversidad y equitatividad se registró en los sitios S3 y S4. La mayor riqueza de especies se observó en el sitio S1 (13), donde se registraron los valores más bajos de diversidad y equitatividad. La abundancia promedio de poliquetos para el área de estudio fue de 546,62 ind. $\mathrm{m}^{-2}(\mathrm{DS}=917,8)$. La mayor abundancia se registró en el Sitio S1, debido a la presencia del poliqueto Fabricinuda sp. que forma estructuras biogénicas de gran extensión con alta densidad de organismos (26826 ind. $\mathrm{m}^{-2}$; DS=13954) (Foto 1). 

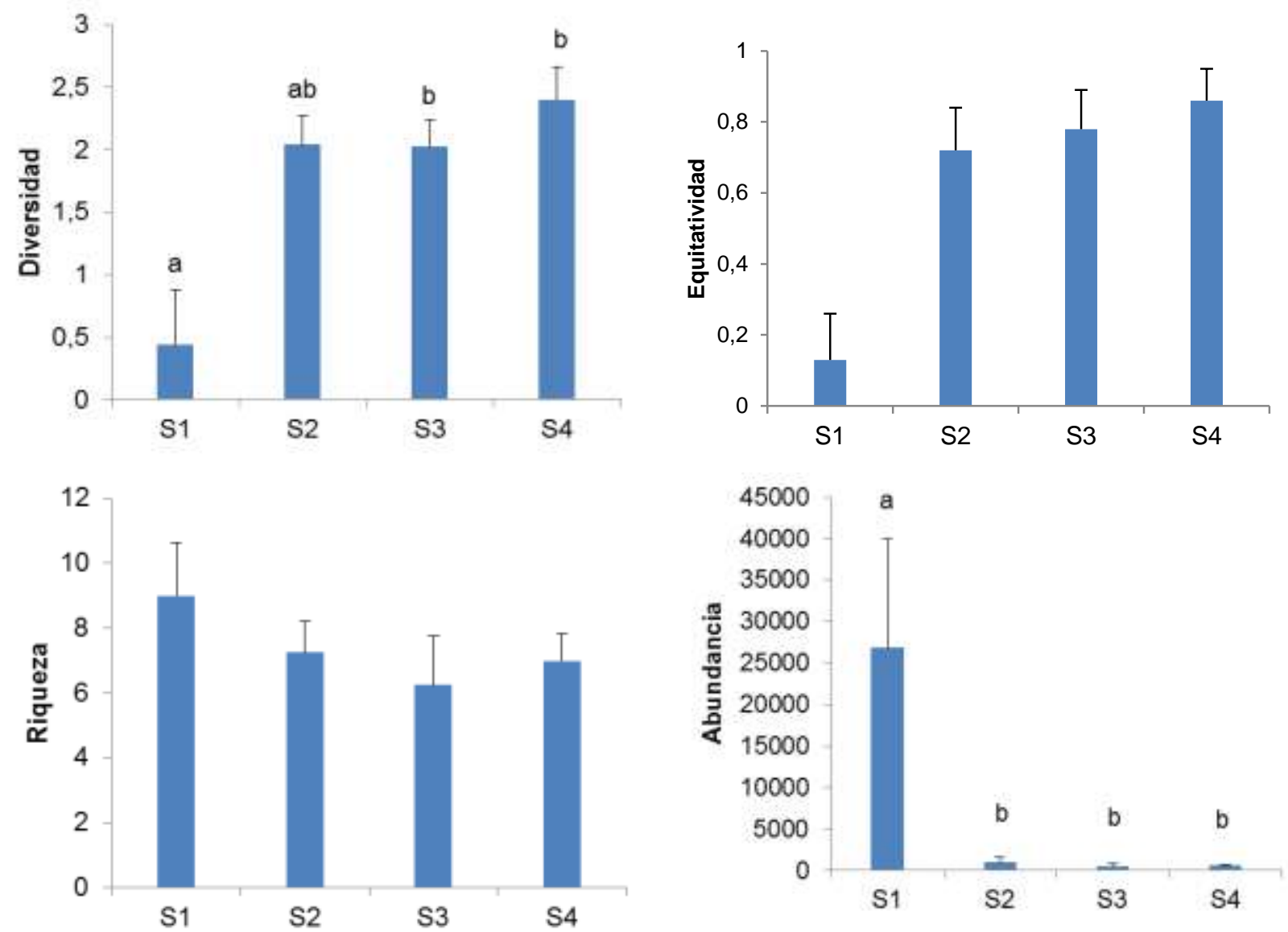

Figura 4. Promedio ( \pm desvío estándar) para los índices calculados por sitio. Medias con una letra común no son significativamente diferentes (Test de Kruskall-Wallis p>0,05).

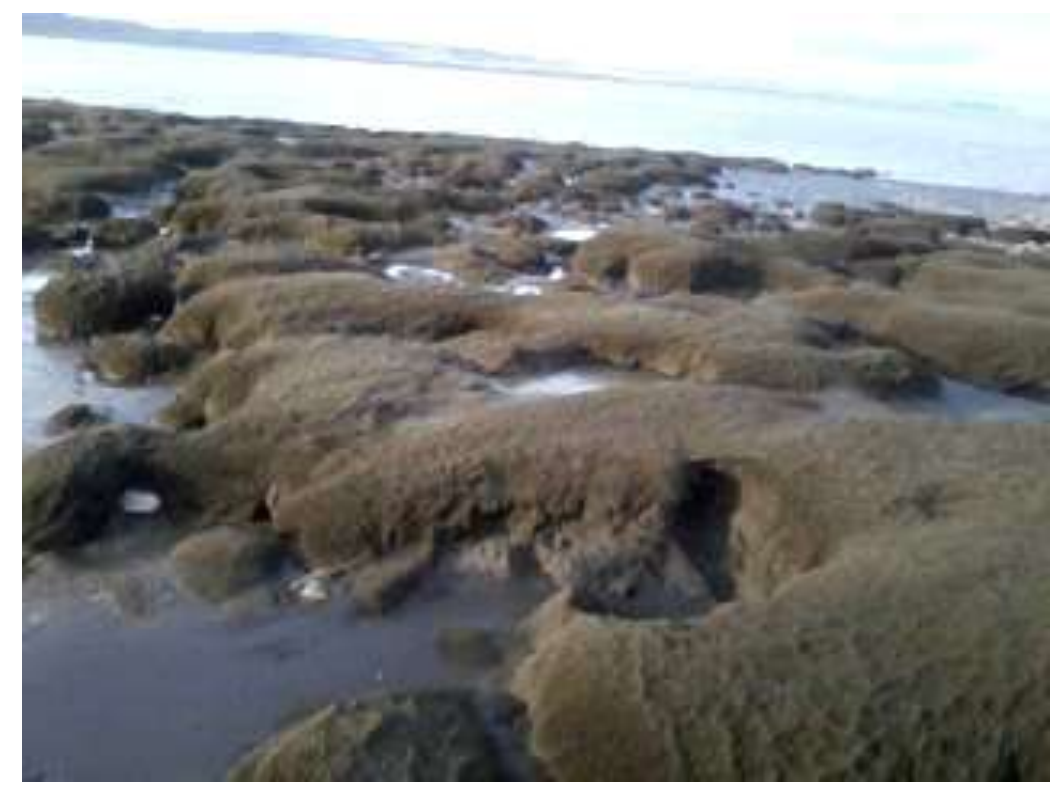

Foto 1. Vista general de las estructuras biogénicas del poliqueto Fabricinuda sp. presente en el sitio S1 (Costanera) del estuario del río Gallegos.

Los resultados del análisis multivariado mostraron diferentes agrupamientos de muestras según distintos niveles de similitud (Fig. 5). El análisis SIMPER permitió identificar a las 
especies que más contribuyeron a la similitud entre las muestras dentro de los diferentes grupos y subgrupos (Tabla 2).

Según un nivel de similitud del $45 \%$, se identificaron dos agrupamientos principales de muestras (Test ANOSIM, R Global =0,921; $\mathrm{p}=0,001$ ). El Grupo A estuvo formado por las muestras del sitio S1, caracterizadas por Fabricinuda sp., Notocirrus lorum, Scolecolepides uncinatus y Kinbergonuphis dorsalis. El Grupo B se encontró integrado por las muestras de los sitios S2, S3 y S4, caracterizadas por Notocirrus lorum, Eteone sculpta, Scoloplos cirratus, Kinbergonuphis dorsalis, Aglaophamus praetiosus, Glycinde armata y Lumbrineris cingulata.

Según un nivel de similitud del 65\%, en el Grupo B se pudieron identificar dos subgrupos (Test ANOSIM, R Global = 0,751; $\mathrm{p}=0,001)$ : $\mathrm{G} 1$ integrado por muestras del sitio S2, caracterizadas por Kinbergonuphis dorsalis, Eteone sculpta, Notocirrus lorum y Scoloplos cirratus, y G2 integrado por muestras de los sitios S3 y S4, caracterizadas por Aglaophamus praetiosus, N. lorum, Glycinde armata y $S$. cirratus.

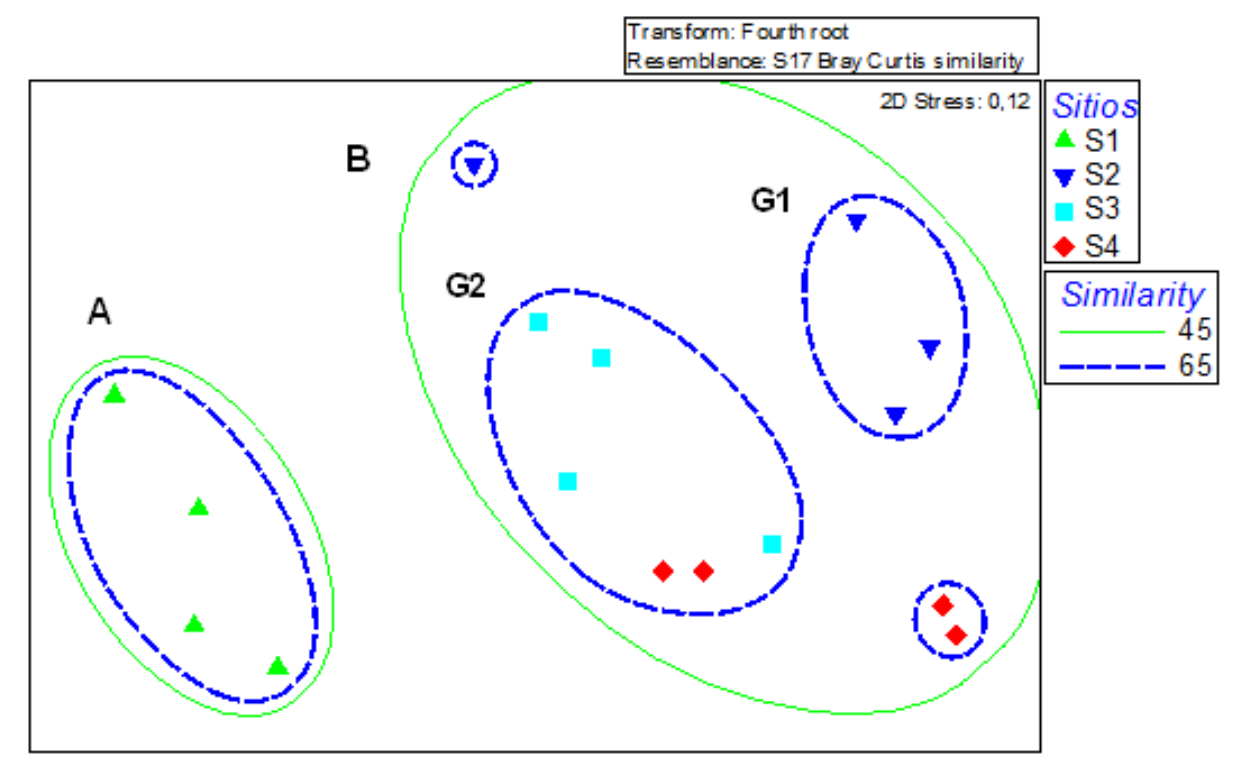

Figura 5. Resultado del análisis de ordenamiento (MDS) y agrupamiento de muestras (análisis de clúster, líneas de color verde y azul), utilizando transformación raíz cuarta de la abundancia, índice de similitud de Bray-Curtis y ligamiento promedio. 
Tabla 2. Análisis SIMPER. Principales especies que contribuyeron a la similitud entre muestras dentro de los grupos y subgrupos. $\mathrm{AP}=$ abundancia promedio (datos transformados por raíz cuarta); $\mathrm{SP}=$ similitud promedio; $\mathrm{DE}=$ desvío estándar; $\mathrm{CP}=$ contribución (\%); $\mathrm{PA}=$ porcentaje acumulado.

\begin{tabular}{|c|c|c|c|c|c|c|c|}
\hline Grupos & Subgrupo & Especies & AP & SP & DE & $\mathbf{C P}$ & PA \\
\hline \multirow{6}{*}{$\begin{array}{c}\text { A } \\
\text { Similitud: } \\
71,62 \%\end{array}$} & & Fabricinuda sp. & 13,81 & 29,72 & 4,54 & 41,5 & 41,51 \\
\hline & & Notocirrus lorum & 4,12 & 8,92 & 2,81 & 12,45 & 53,95 \\
\hline & & $\begin{array}{l}\text { Scolecolepides } \\
\text { uncinatus }\end{array}$ & 3,43 & 8,31 & 8,37 & 11,61 & 65,56 \\
\hline & & $\begin{array}{l}\text { Kinbergonuphis } \\
\text { dorsalis }\end{array}$ & 3,66 & 7,63 & 7,11 & 10,65 & 76,21 \\
\hline & & Glycinde armata & 3,08 & 6,91 & 3,39 & 9,65 & 85,86 \\
\hline & & Eteone sculpta & 2,86 & 5,73 & 3,41 & 7,99 & 93,85 \\
\hline \multirow{13}{*}{$\begin{array}{c}\text { B } \\
\text { Similitud: } \\
61,46 \%\end{array}$} & \multirow{6}{*}{$\begin{array}{c}\text { G1 } \\
\text { Similitud: } \\
62,49 \%\end{array}$} & $\begin{array}{l}\text { Kinbergonuphis } \\
\text { dorsalis }\end{array}$ & 3,83 & 15,53 & 3,15 & 24,84 & 24,84 \\
\hline & & Eteone sculpta & 3,02 & 12,95 & 2,77 & 20,72 & 45,57 \\
\hline & & Notocirrus lorum & 2,38 & 10,39 & 5,4 & 16,63 & 62,2 \\
\hline & & Scoloplos cirratus & 2,62 & 9,21 & 2,18 & 14,74 & 76,93 \\
\hline & & Clymenella minor & 1,94 & 5,93 & 0,87 & 9,49 & 86,43 \\
\hline & & Glycinde armata & 2,14 & 4,45 & 0,91 & 7,13 & 93,55 \\
\hline & \multirow{7}{*}{$\begin{array}{c}\text { G2 } \\
\text { Similitud: } \\
66,24 \%\end{array}$} & $\begin{array}{l}\text { Aglaophamus } \\
\text { praetiosus }\end{array}$ & 2,98 & 14,4 & 4,89 & 21,74 & 21,74 \\
\hline & & Notocirrus lorum & 2,6 & 9,69 & 1,63 & 14,63 & 36,37 \\
\hline & & Glycinde armata & 2,21 & 9,64 & 1,64 & 14,55 & 50,92 \\
\hline & & Scoloplos cirratus & 2,34 & 9,47 & 1,5 & 14,3 & 65,21 \\
\hline & & Eteone sculpta & 2,37 & 7,93 & 0,99 & 11,98 & 77,19 \\
\hline & & $\begin{array}{l}\text { Kinbergonuphis } \\
\text { dorsalis }\end{array}$ & 2,24 & 6,93 & 1,03 & 10,46 & 87,65 \\
\hline & & $\begin{array}{l}\text { Lumbrineris } \\
\text { cingulata }\end{array}$ & 1,88 & 4,8 & 0,72 & 7,25 & 94,9 \\
\hline
\end{tabular}

\section{DISCUSION}

En el intermareal del estuario del río Gallegos se identificaron 14 especies de poliquetos de fondos blandos, pertenecientes a 12 familias, que conformaron distintas asociaciones vinculadas a diferentes características del sedimento. La asociación dominada por Fabricinuda sp., se encontró en sedimentos constituidos principalmente por arena fina y muy fina del sitio S1. De esta asociación participaron otras especies como Notocirrus lorum, Scolecolepides uncinatus, Kinbergonuphis dorsalis, Glycinde armata, Eteone sculpta, Clymenella minor, Ophioglycera eximia y Travisia sp. Esta asociación presentó la mayor riqueza específica, pero la menor diversidad y equitatividad.

La asociación caracterizada por Kinbergonuphis dorsalis, Eteone sculpta, Notocirrus lorum y Scoloplos cirratus se encontró vinculada a sedimentos con alto porcentaje de limo y arcilla (> $50 \%$ ) y mayor contenido de materia orgánica, en el sitio S2, ubicado en la zona de vertido de los efluentes domiciliarios provenientes de la ciudad de Río Gallegos. La asociación caracterizada por Aglaophamus praetiosus, N. lorum, Glycinde armata y $S$. cirratus se encontró en sedimentos con proporciones variables de arena, limo y arcilla de los sitios S3 y S4, más alejados de la ciudad de Río Gallegos y con porcentajes más bajos de materia orgánica que el sitio S2. Varios autores han demostrado que las características del sedimento son los factores primarios que determinan los patrones espaciales de las comunidades del 
macrobentos y de las agrupaciones de poliquetos (Gray, 1974, Carrasco, 1996, 1997; Pastor de Ward, 2000, Hernandez-Arana, 2003, Díaz Castañeda y Harris, 2004; Bremec y Giberto, 2006).

El análisis de los parámetros comunitarios mostró diferencias significativas entre el sitio S1 y los demás sitios estudiados, presentando la mayor abundancia de individuos así como los valores más bajos de diversidad y equitatividad. Esto se debió a la marcada dominancia numérica de Fabricinuda sp. Este poliqueto, se distribuye en el nivel intermareal inferior en sedimentos finos pero con bajas proporciones tanto de materia orgánica como de limosarcillas. En este estudio, se registró exclusivamente en cercanías de la ciudad de Río Gallegos en el sitio S1 y se trata, posiblemente, de una especie no descripta (J. Kirk Fitzhugh, Natural History Museum of Los Angeles County, comunicación personal). En estudios previos, realizados en el mismo sitio, se ha observado que construye estructuras biogénicas conformadas por tubos de arena, con alta densidad de individuos, constituyendo un hábitat particular donde la riqueza de especies y la abundancia de organismos bentónicos son más altas que en sitios adyacentes sin su presencia (Pittaluga, 2016, Lizarralde et al., 2018). Además, tres especies de moluscos bivalvos (Sphenia hatcheri, Perumytilus purpuratus y un bivalvo indeterminado) y una especie de poliqueto (Ophioglycera eximia) se asocian exclusivamente con las estructuras biogénicas de Fabricinuda sp. (Lizarralde et al., 2018). Por otra parte, se ha señalado que la rápida secreción de nuevos tubos y la capacidad de los poliquetos Fabriciidae de emigrar de ellos cuando se deterioran las condiciones ambientales, podrían ser adaptaciones a entornos inestables que les permitirían colonizar rápidamente nuevos ambientes (Knight-Jones, 1981; Giangrande et al., 2014).

En el nivel superior de los sitios S1 y S3 y en el inferior del S2, se registró la presencia del poliqueto Scolecolepides uncinatus asociado con sedimentos limo-arcillosos y con altos contenidos de materia orgánica. $S$. uncinatus ha sido señalado previamente como dominante en sedimentos limo-arcillosos del sitio S1 (Costanera) por Lizarralde y Pittaluga (2011), así como en sitios areno-fangosos de Bahía Loma en el estrecho de Magallanes (Cañete et al., 2010). Algunos poliquetos de la familia Spionidae, a la cual pertenece $S$. uncinatus han sido descriptos por numerosos autores (Pearson y Rosenberg, 1978; Bigot et al., 2006; Vivan et al., 2009), como organismos oportunistas, y han sido empleados como bioindicadores para monitorear el impacto producido por el enriquecimiento de materia orgánica.

En este trabajo se describe la composición y distribución de la fauna de poliquetos del estuario del río Gallegos, así como las características sedimentológicas del ambiente, sin embargo, se considera importante continuar con monitoreos en el área, e introducir como variables aquellas que permitan comparar sitios con diferente impacto antrópico, sobre todo en la margen sur donde se asienta la ciudad de río Gallegos. Esto permitirá evaluar la importancia de estos organismos como indicadores de perturbaciones ambientales o antrópicas, y seleccionar aquellas especies que pudieran emplearse como bioindicadoras.

\section{CONCLUSIONES}

Se identificaron 14 especies de poliquetos en fondos blandos del ambiente intermareal del estuario del río Gallegos.

Las especies más abundantes fueron: Fabricinuda sp., que se encontró asociada con el nivel inferior del intermareal en sitios con predominio de arenas finas, seguido por Notocirrus lorum y Kinbergonuphis dorsalis que se distribuyeron en niveles medios y bajos en sedimentos limo-arcillosos. 
Se reconocieron tres asociaciones principales de poliquetos, vinculadas a sedimentos con diferente contenido de materia orgánica y distinta composición granulométrica.

\section{BIBLIOGRAFIA}

BASTIDA, R., ZAMPONI, M., BREMEC, C., ROUX, A., GENZANO, G. y ELÍAS, R. (2007). Las comunidades bentónicas. En Carreto, J.I. y Bremec C. (eds) El Mar Argentino y sus recursos pesqueros, 5: 91-125.

BIGOT, L., CONAND, C., AMOUROUX, J., FROUIN, P., BRUGGEMANN, H. у GREMARE, A. (2006). Effects of industrial outfalls on tropical macrobenthic sediment communities in Reunion Island (Southwest Indian Ocean). Marine Pollution Bulletin 52:865-880. https://doi.org/10.1016/j.marpolbul.2005.11.021

BREMEC, C.S. (1986). Asociaciones del macrobentos infralitoral de Monte Hermoso (39 00' S-61ำ' W, República Argentina). Spheniscus, Bahía Blanca, 2: 1-18.

BREMEC, C. y GIBERTO D. (2006). Polychaete assemblages in the Argentinean Biogeographical Province, between $34^{\circ}$ and $38^{\circ}$ S. Sci. Mar. 70(S3): 249-257. https://doi.org/10.3989/scimar.2006.70s3249

CAÑETE, J.I., G. LEIGHTON y SOTO, E. (2000). Proposición de un índice de vigilancia ambiental basado en la variabilidad temporal de la abundancia de dos especies de poliquetos bentónicos de Bahía Quintero, Chile. Revista de Biología Marina y Oceanografía 35: 185-194. https://doi.org/10.4067/s0718-19572000000100007

CAÑETE, J.I., ASTORGA, M.S., SANTANA, M. y PALACIOS, M. (2010). Abundancia y distribución espacial de Scolecolepides uncinatus Blake, 1983 (Polychaeta: Spionidae) y características sedimentológicas en Bahía Lomas, Tierra del Fuego, Chile. Anales Instituto Patagonia (Chile), 38(2):81-94. https://doi.org/10.4067/s0718686x2010000200008

CARRASCO, F. D. (1996). The benthic macroinfauna of Concepcion Bay, Chile: High ecological dominance at the shallow sublittoral off Lirquen. Gayana Oceanol., 4 (1): $1-12$.

CARRASCO, F. D. (1997). Sublittoral macrobenthic fauna off Punta Coloso, Antofagasta, northern Chile: High persistence of the polychaete assemblage. Bull. Mar. Sci., 60 (2): 443-459. https://doi.org/10.3989/scimar.2006.70s3169

CODIGNOTTO, J.A. (1990). Evolución en el cuaternario alto del sector de costa y plataforma submarina entre río Coig, Santa Cruz y Punta María Tierra del Fuego. Asociación Geológica Argentina, 45: 19-16.

CLARKE K.R. y WARWICK R.M. (2001). Change in marine communities: an approach to statistical analysis and interpretation. PRIMER-E, Plymouth, $159 \mathrm{pp}$.

DAUVIN, J.C., RUELlETA, T., DESROYA, N. y JANSON, A.L. (2007).The ecological quality status of the Bay of Seine and the Seine estuary: Use of biotic indices. Marine Pollution Bulletin. Vol 6, 1-9. https://doi.org/10.1016/j.marpolbul.2006.04.010

DÍAZ-CASTANEDA, V. M. y HARRIS, L. (2004). Biodiversity and structure ofthe polychaete fauna from soft-bottoms of Bahía Todos Los Santos, Baja California, Mexico. Deep Sea Research Part II: Topical Studies in Oceanography, 51, 827-847. https://doi.org/10.1016/j.dsr2.2004.05.007

ELÍAS, R. y BREMEC C. (1986). Macrobentos del área de la bahía Blanca (Argentina). II. Relaciones entre asociaciones de sustrato móvil. Spheniscus, Bahía Blanca, 3: 51-52. https://doi.org/10.1590/s1679-87591990000200001

ELÍAS, R. (1992). Inventario del macrobentos infaunal de la Bahía Blanca. I: Poliquetos. Neotropica, 38 (100): 8695. 
ELÍAS, R. y IENO E.N. (1993). La comunidad de Laeonereis acuta Treadwell,1923

(Polychaeta: Nereididae) en la región interna de la Bahía Blanca. Iheringia, Ser. Zool. 75: 3-13.

ELÍAS, R., JAUBET, M.L, FERRANDO, A. y SARACHO BOTTERO M.A. (2017). Historia y perspectivas de los estudios sobre poliquetos en Argentina 1: 3-23 En: Poliquetos de Sudamérica. Díaz-Díaz, O., D. Bone, C.T. Rodríguez y V.H. DelgadoBlas (Eds.). Poliquetos de Sudamérica. Volumen especial del Boletín del Instituto Oceanográfico de Venezuela. Cumaná, Venezuela 149 pp. https://doi.org/10.15517/rbt.v54i3.12775

FERRARI, S., ALBRIEU, C. y GANDINI, P. (2002). Importance of the Rio Gallegos estuary, Santa Cruz, Argentina, for migratory shorebirds. Wader Study Group Bull, 99: 35 40.

FERRARI, S., ERCOLANO, B. y ALBRIEU, C. (2007). Pérdida de hábitat por actividades antrópicas en las marismas y planicies de marea del estuario del río Gallegos (Patagonia austral, Argentina). 319-327 pp. En Castro Lucic, M y Fernández Reyes, L. (eds.) Gestión Sostenible de Humedales. Editorial CYTED y Programa Internacional de Interculturalidad, Santiago de Chile. https://doi.org/10.18356/99b19ee0-es

GAMBI, M.C., CONTI, G. y BREMEC, C.S. (1998). Polychaete distribution, diversity and seasonality related to seagrass cover in shalow soft bottoms of the Tyrrhenuan Sea (Italy). Sci. Mar. 62 (1 2): 1-17. https://doi.org/10.3989/scimar.1998.62n1-21

GIANGRANDE A., M. LICCIANO y MUSCO, L. (2005). Polychaetes as Environmental Indicators Revisited. Marine Pollution Bulletin 50 (11): 1153-62. https://doi.org/10.1016/j.marpolbul.2005.08.003

GIANGRANDE, A., GAMBI, M.C., MICHELI, F. y KROEKER, K.J. (2014). Fabriciidae (Annelida, Sabellida) from a naturally acidified coastal system (Italy) with description of two new species. Journal of the Marine Biological Association of the United Kingdom, 94(7): 1-11. https://doi.org/10.1017/s0025315414000678

GRAY, J.S., MCINTYRE, A. y STIRN, J. (1992). Manual of methods in aquatic environment research. FAO Fisheries Technical Paper $N^{\circ} 11$. Roma, Italia. 49 pp.

GRAY J.S. (1974). Animal-sediment relationships. Oceanogr. Mar. Biol. Annu. Rev. 12: 223-261.

HERNÁNDEZ-ARANA, H.A., ROWDEN, A.A., ATTRILL, M., WARWICK, R. y GOLDBOUCHOT, G. (2003). Large-scale environmental influences on the benthic macroinfauna of the southern Gulf of Mexico. Estuar. Coast. Shelf Sci., 58: 825-841. https://doi.org/10.1016/s0272-7714(03)00188-4

KNIGHT-JONES, P. (1981). Behaviour, chaetal inversion and phylogeny of Sabellida (Polychaeta). Zoologica Scripta 10:183-202. https://doi.org/10.1111/j.14636409.1981.tb00495.x

LIZARRALDE Z.I. y PITTALUGA S. (2011). Distribution and temporal variability of the benthic fauna in a tidal flat of the Rio Gallegos Estuary, Patagonia, Argentina. Thalassas 27: 9-20. https://doi.org/10.1007/s41208-017-0045-y

LIZARRALDE Z.I., PITTALUGA, S. y PERRONI, M. (2018). Changes of Benthic Macrofaunal Composition on a Tidal Flat of Río Gallegos Estuary, Argentina. Thalassas 34: 131-138. ISSN 0212-5919. https://doi.org/10.1007/s41208-017-0045-y

LIZARRAlDE, Z., FERRARI, S., PITTALUGA, S. y ALBRIEU, C. (2010). Seasonal abundance and trophic ecology of Hudsonian Godwit (Limosa haemastica) at Río Gallegos estuary Patagonia, Argentina. Ornitología Neotropical, 21: 283-294. 
MARTIN, J.P., BASTIDA, R. y TRASSENS, M. (2004). Polychaetes assemblages of intertidal mixohaline flats of Bahía Samborombón (La Plata River Estuary, Argentina). Thalassas 20 (2): 39-53.

MARTIN, J.P. y BASTIDA R. (2006). Life history and reproduction of Capitella "capitata" (Capitellidae: Polychaeta) in Río de La Plata Estuary (Argentina). Thalassas 22: 25-38

MARTIN J.P., BASTIDA R. y TRASSENS, M. (2009). Estudio exploratorio para la detección de bioindicadores de impacto ambiental en la Ría Deseado (Patagonia austral, Argentina). VII JNCM, Bahía Blanca.

MARTIN J.P., SAR A. y CAMINOS, C. (2015). Variaciones espacio-temporales en la comunidad infaunal de planicies arenofangosas de la Bahía de San Julián (Patagonia austral, Argentina). IX JNCM, Ushuaia.

PASTOR DE WARD, C.T. (2000). Polychaete assemblages in the San José Gulf (Chubut, Argentina) in relation to abiotic and biotic factors. P.S.Z.N.I. Mar. Ecol., 21: 175-190. https://doi.org/10.1046/j.1439-0485.2000.00704.x

PEARSON, T. H. y ROSENBERG, R. (1978). Macrobenthic succession in relation to organic enrichment and pollution of the marine environment. Oceanogr. Mar. Biol. Annu,16: 229311.

PICOLLO, M.C. y PERILLO, G.M. (1997). Geomorfología e hidrografía de los estuarios. Pp 133-161 en Boschi, E. (eds.) El Mar argentino y sus recursos pesqueros. Editorial INIDEP, Mar del Plata.

PITTALUGA, S. (2016). Caracterización de las comunidades bentónicas de fondos blandos del estuario del río Gallegos: su utilización en la detección de impactos antrópicos. Tesis de Maestría en Manejo y Gestión de Recursos Naturales en Patagonia (UNPA), Río Gallegos. https://doi.org/10.22305/ict-unpa.v10i1.263

POCKLINGTON P. y WELLS, P.G. (1992). Polychaetes: key taxa for marine environmental quality monitoring. Mar. Poll. Bull. 24:59-598. https://doi.org/10.1016/0025326x(92)90278-e

RINGUELET, R. (1963). Estudio ecológico en el litoral patagónico. El piso supralitoral en la ría Deseado (Santa Cruz, Argentina). Physis, 24 (67): 103-106.

RINGUELET, R., DIONI, W. y BUCKLE, F. (1963). Reconocimiento previo de la distribución de la iliofauna en el fango intertidal de Puerto Deseado (Santa Cruz, Argentina). Physis, 24 (67): 95-101.

RINGUELET, R., AMOR, A., MAGALDI, N. y PALLARES, R. (1962). Estudio ecológico de la fauna intercotidal de Puerto Deseado, en febrero de 1961 (Santa Cruz, Argentina). Physis, 23 (64): 35-53.

SCHULTE, E.E. y HOPKINS, B.G. (1996). Estimation of organic matter by weight loss-onignition. Pp 21-31 in Magdoff, F.R et al. (eds.) Solid organic matter: Analysis and interpretation. SSSA Special Publication 46. SSSA, Madison, WI. https://doi.org/10.2136/sssaspecpub46.c3

VIVAN, J.M., DI DOMENICO, M. y MARQUES DE ALMEIDA, T.C. (2009). Effects of dredged material disposal on benthic macrofauna near Itajaí Harbour (Santa Catarina, south Brazil). Ecological Engineering 35:1435-1443. https://doi.org/10.1016/j.ecoleng.2009.06.005

ZAR, J.H. (1996.) Biostatistical analysis. 3 ed. Prentice-Hall International, Englewood Cliffs, New Jersey, US. 